\title{
PARTICLE ACCELERATION AND NONTHERMAL PHENOMENA IN SUPERBUBBLES
}

\author{
ANDREI M. BYKOV \\ A.F. Ioffe Institute for Physics and Technology, 194021, St. Petersburg, Russia
}

(Received: 22 July 2000; Accepted in final form: 24 November 2000)

\begin{abstract}
Models of nonthermal particle acceleration in the vicinity of active star forming regions are reviewed. We discuss a collective effect of both stellar winds of massive stars and core collapsed supernovae as particle acceleration agents. Collective supernova explosions with great energy release in the form of multiple interacting shock waves inside the superbubbles are argued as a favourable site of nonthermal particle acceleration. The acceleration mechanism provides efficient creation of a nonthermal nuclei population with a hard low-energy spectrum, containing a substantial part of the kinetic energy released by the winds of young massive stars and supernovae. We discuss a model of temporal evolution of particle distribution function accounting for the nonlinear effect of the reaction of the accelerated particles on the shock turbulence inside the superbubble. The model illustrates that both the low-energy metal-rich nonthermal component and the standard galactic cosmic rays could be efficiently produced by superbubbles at different evolution stages.
\end{abstract}

\section{Introduction}

Young massive stars formation is known to be spatially and temporally correlated with OB associations. Massive star formation occurs in massive molecular clouds (e.g., Blitz, 1993). The most massive O stars begin to explode as core collapsed supernovae about a million years after the formation of an OB association, creating a superbubble (SB) filled with hot tenuous plasma with supersonic turbulence. Bright X-ray emission has been observed from the hot gas in superbubbles in the Large Magellanic Cloud (LMC). An attempt to identify primary SNR shocks in SB interior has been made by Chen et al. (2000). They used Hubble Space Telescope WFPC2 emission-line ( $\mathrm{H} \alpha$ and [S II] lines) images of three SBs in the LMC to identify SNR shocks inside the superbubbles. Such strong and moderate strength SNR shocks could be attributed to filamentary nebular morphology seen in some SBs. From the other hand numerous weak shocks expected in the hot tenuous superbubble interiors are not producing optical signatures and can hardly be observed with such a technique.

There are some HI, IR, radio and X-ray evidences for the presence of several supershells and SBs within a local kpc from the Sun. The most impressive local supershell GSH $238+00+09$ with the mass $\sim 2.7 \times 10^{6} M_{\odot}$ and radius $\sim 220 \mathrm{pc}$ at the distance $\sim 0.8 \mathrm{kpc}$ has been found by Heiles (1998). He estimated the energy required to produce such a shell as $3.4 \times 10^{52} \mathrm{erg}$, that implies some 30 supernovae 
to be involved. The kinematic age of the superbubble is about $10 \mathrm{Myr}$. From the standard Salpeter's stellar initial mass function (IMF) and nucleosynthetic yields of exploded massive stars (e.g., Woosley and Weaver, 1995) a metallicity of the hot SB interior at the current stage of $\gtrsim 2 Z_{\odot}$ is derived. The hot SB gas metallicity may have been as high as $\gtrsim 10 Z_{\odot}$ at earlier stages of the SB evolution. Being supplied with kinetic energy from extremely powerful sources such as core collapsed supernovae and winds of massive early type stars, SBs should be very plausible sites of nonthermal particle acceleration (Bykov and Fleishman, 1992; Bykov, 1995; Parizot, 1998; Higdon et al., 1998). The SBs in the local vicinity must be taken into account in the cosmic ray (CR) propagation modeling.

The structures of velocity, density and magnetic fields in a SB are rather complicated due to discrete nature of energy and momentum sources which is important during the first few million years as well as due to the interactions of the parent molecular cloud with winds and shocks. Direct observational data on the MHD motions of hot tenuous gas inside the SB are rather scarce yet. The shock turbulence formation inside the SB should occur due to multiple interactions of the shocks with the clouds following the models suggested by Bykov and Toptygin (1987) and Bykov (1988). Simulations of 3D global dynamics of SBs in the interstellar medium (ISM) with account of the effect of the ISM magnetic field and ISM stratification have been performed by Tomisaka (1998). Korpi et al. (1999) simulated the 3D dynamics of a SB accounting for inhomogeneous ISM structure and large scale ISM turbulence. These simulations assume continuous momentum supply from the OB star winds and supernovae as mechanical luminosity and do not resolve supersonic MHD turbulent motions inside the SB that are important for nonthermal particle production. Shocks and MHD turbulent motions inside a SB can efficiently transfer their energy to CRs because the timescale of particle acceleration in a SB is below Myr and the efficiency of energy conversion could be above $\gtrsim 30 \%$ at least during the first $3 \mathrm{Myr}$ of the SB evolution (Bykov, 1999). Recent global models of SB evolution are based on nonrelativistic one-component perfect gas law inside the SB (e.g., Tomisaka, 1998; Korpi et al., 1999). The effect of $\mathrm{CR}$ acceleration inside a SB would make the gas specific heat ratio to be closer to $4 / 3$ and provide effective energy leakage from SB interiors due to escaping of fast particles even before the radiative stage. These effects could be important for simulations of the global dynamics of SBs.

\section{Particle Acceleration in Superbubbles}

To construct a model of nonthermal particle evolution in the vicinity of star forming regions the following assumptions were taken by Bykov (1995):

(1) Particle acceleration is produced by a powerful energy release in the form of violent plasma motions which occur in a bubble created by stellar winds and $\mathrm{SNe}$. The bubble is filled with hot rarefied plasma of enhanced metallicity. 
(2) The nonthermal particles accelerated within the bubble then penetrate into the dense matter (a supershell or a cloud) surrounding the bubble. The particles suffer Coulomb and nuclear interactions with the dense matter which leads to nonthermal emission, nucleosynthesis and spallation reactions, and $\gamma$-ray production. Particle reacceleration by MHD turbulence inside the supershell is important and could compensate the Coulomb losses (cf., Seo and Ptuskin, 1994).

\subsection{Spectra of the Nonthermal Particles}

The energy gain of the suprathermal nuclei injected into the bubble occurs due to large scale MHD motions of magnetized plasma.

A kinetic energy release within the bubble created by a stellar association may reach a few times $10^{38} \mathrm{erg} \mathrm{s}^{-1}$ at the stages of intense stellar winds and multiple $\mathrm{SN}$ explosions. The process is accompanied by formation of shocks, large scale flows and broad spectra of MHD fluctuations in a tenuous plasma with frozenin magnetic fields. Vortex electric fields generated by the large scale motions of highly conductive plasma with shocks result in a non-equilibrial distribution of the charged nuclei. The particle distribution within such a system is highly intermittent. Statistical description of intermittent systems differs from the description of homogeneous systems (Bykov and Toptygin, 1993).

The distribution function $N(\mathbf{r}, p, t)$ of nonthermal nuclei (with energies up to $\mathrm{GeV}$ range) averaged over an ensemble of turbulent motions and shocks satisfies the kinetic equation

$$
\frac{\partial N}{\partial t}-\frac{\partial}{\partial r_{\alpha}} \chi_{\alpha \beta} \frac{\partial N}{\partial r_{\beta}}=G \hat{L} N+\frac{1}{p^{2}} \frac{\partial}{\partial p} p^{4} D \frac{\partial N}{\partial p}+A \hat{L}^{2} N+2 B \hat{L} \hat{P} N+F_{j}(p),
$$

The source term $F_{j}(p)$ is determined by injection of the nuclei of a type $j$. The integro-differential operators $\hat{L}$ and $\hat{P}$ are given by

$$
\hat{L}=\frac{1}{3 p^{2}} \frac{\partial}{\partial p} p^{3-\gamma} \int_{0}^{p} \mathrm{~d} p^{\prime} p^{\prime \gamma} \frac{\partial}{\partial p^{\prime}} ; \quad \hat{P}=\frac{p}{3} \frac{\partial}{\partial p} .
$$

The kinetic coefficients $A, B, D, \tau_{\mathrm{sh}}$, and $\chi_{\alpha \beta}$ are expressed in terms of the spectral functions that describe correlations between large scale turbulent motions and shocks, the index $\gamma$ depends on the shock ensemble properties (Bykov and Toptygin, 1993). The kinetic coefficients satisfy the following renormalization equations:

$$
\begin{aligned}
& \chi=\kappa+\frac{1}{3} \int \frac{\mathrm{d}^{3} \mathbf{k} \mathrm{d} \omega}{(2 \pi)^{4}}\left[\frac{2 T+S}{i \omega+k^{2} \chi}-\frac{2 k^{2} \chi S}{\left(i \omega+k^{2} \chi\right)^{2}}\right], \\
& D=\frac{\chi}{9} \int \frac{\mathrm{d}^{3} \mathbf{k d} \omega}{(2 \pi)^{4}} \frac{k^{4} S(k, \omega)}{\omega^{2}+k^{4} \chi^{2}}, \\
& A=\chi \int \frac{d^{3} \mathbf{k} \mathrm{d} \omega}{(2 \pi)^{4}} \frac{k^{4} \tilde{\phi}(k, \omega)}{\omega^{2}+k^{4} \chi^{2}},
\end{aligned}
$$




$$
B=\chi \int \frac{\mathrm{d}^{3} \mathbf{k} \mathrm{d} \omega}{(2 \pi)^{4}} \frac{k^{4} \tilde{\mu}(k, \omega)}{\omega^{2}+k^{4} \chi^{2}} .
$$

Here $G=\left(1 / \tau_{\mathrm{sh}}+B\right) . T(k, \omega)$ and $S(k, \omega)$ are the transverse and longitudinal parts of the Fourier components of the turbulent velocity correlation tensor. Correlations between velocity jumps on shock fronts are described by $\tilde{\phi}(k, \omega)$, while $\tilde{\mu}(k, \omega)$ represents shock-rarefaction correlations. The introduction of these spectral functions is dictated by the intermittent character of the system with shocks.

The test particle calculations of Bykov and Fleishman 1992) have shown that the low energy branch of the particle distribution would contain a substantial fraction of the free energy of the system after a few acceleration times. Thus, to calculate the efficiency of the shock turbulence power conversion to the nonthermal particle component as well as the particle spectra we have to account for the backreaction of the accelerated particles on the shock turbulence. To do that we supplied the kinetic equations (1)-(6) with the energy conservation equation for the total system including the shock turbulence and the nonthermal particles. We also used the simplified equations for the large scale shock turbulence spectral functions (Bykov, 1988) including the rate $\gamma_{\mathrm{cr}}$ of longitudinal turbulence $(S(k, t))$ damping due to particle acceleration (cf., Equation (4)).

$$
\begin{aligned}
& \frac{\partial S(k, t)}{\partial t}-\frac{\partial \Pi_{\alpha}^{s}(S, k, t)}{\partial k_{\alpha}}=\gamma_{v s} T-\gamma_{\mathrm{cr}} S-\gamma_{d s} S, \\
& \frac{\partial T(k, t)}{\partial t}-\frac{\partial \Pi_{\alpha}^{v}(T, k, t)}{\partial k_{\alpha}}=\gamma_{v v} T-\gamma_{v s} T-\gamma_{d v} T .
\end{aligned}
$$

The mode energy transformation rates $\gamma_{v s}, \gamma_{d s}, \gamma_{v v}, \gamma_{d v}$ as well as the turbulence nonlinear cascade spectral fluxes $\Pi_{\alpha}^{s}(S, k, t), \Pi_{\alpha}^{v}(T, k, t)$ are described in Bykov (1988).

The energy losses of the accelerated nuclei inside the bubble filled with very rarefied plasma are relatively unimportant and they are neglected in Equation (1). Equation (1) takes into account particle acceleration by shocks and large scale plasma motions as well as resonant particle acceleration by small scale MHD turbulence.

In that model we fixed the scale $R$ of a SB at any particular time. This parameterisation is possible because the particle acceleration time inside a hot SB is much shorter than the SB expansion time $\tau_{\exp } \sim R / \dot{R}$ that is determined by a relatively low velocity of the massive supershell.

Figure 1 shows the calculated efficiency of turbulence energy transfer to nonthermal particles for a SB of a scale $R=220 \mathrm{pc}$. We assumed continuous injection of monoergetic nuclei with the injection energy loading parameter $\zeta=10^{-3}$. Figure 1 (left panel) illustrates the case of injection of nonrelativistic particles of a momentum $p_{0}$, i.e., $F(p) \propto \delta\left(p-p_{0}\right)$. In our particular case the momentum $p_{0}$ corresponds to the energy of $10 \mathrm{keV} \mathrm{amu}{ }^{-1}$. In Figure 2 (left panel) we show the distribution function (normalized $\propto p^{2} N$ ) calculated for that case. 


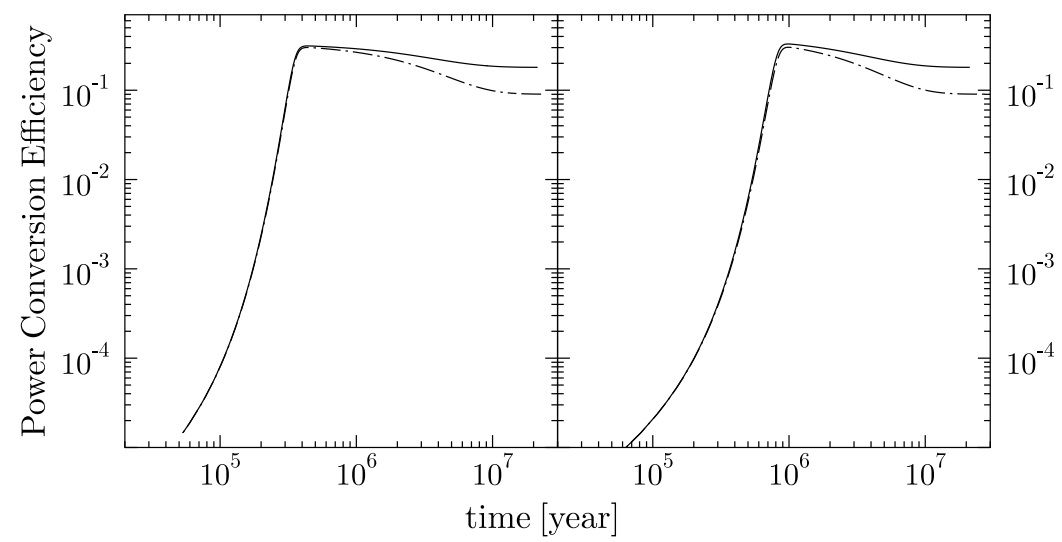

Figure 1. The temporal evolution of the power conversion efficiency to the nonthermal nuclei for two SB scale sizes $R=220 \mathrm{pc}$ (solid lines) and $R=100 \mathrm{pc}$ (dot-dashed lines). Both nonrelativistic (left panel) and relativistic (right panel) particle injection regimes are shown.

It should be noted here that since the nonlinear effects were taken into account in the model the distribution function calculated for monoenergetic injection must not have any of the general properties of the Green function of a linear system. Thus one can not anymore construct the distribution function in the nonlinear case using the superposition principle. That is a very serious compication of the models with efficient particle acceleration. Figures 1 and 2 (right panels) illustrate the efficiency and temporal evolution of the particle spectra for the same SB as described above, but with injection of relativistic monoergetic nuclei of $p_{0 \mathrm{r}}$ corresponding to the energy of $10 \mathrm{GeV} \mathrm{nucl}^{-1}$. From Figure 2 one can see that in both cases (nonrelativistic and relativistic injection) the time asymptotic of the distribution function is a real power-law. In the nonrelativistic case it has a slope close to 3, while in the relativistic case it is closer to 2 .

\subsection{Nonthermal COMPONEnt COMPosition}

At the early stage of a SB evolution the elemental abundances inside a SB filled with a rarefied hot gas can differ strongly from the standard cosmic abundances due to ejection of matter enriched with some heavy elements from $\mathrm{SNe}$ and stellar winds of massive stars of (WR and OB type).

We can indicate the two most important injection processes in that model (Bykov, 1995).

(1) Creation of suprathermal nuclei by collisionless shock waves within a hot bubble. The injection produced directly by a collisionless shock depends on the rigidity. One may expect that the injection of $\mathrm{O}, \mathrm{C}, \mathrm{Ne}, \mathrm{Mg}$, Si nuclei (as well as of other nuclei with $A / Q=2$ ) in a hot plasma of the bubble has the same efficiency as the injection of $\alpha$-particles in a hydrogen-helium plasma. 


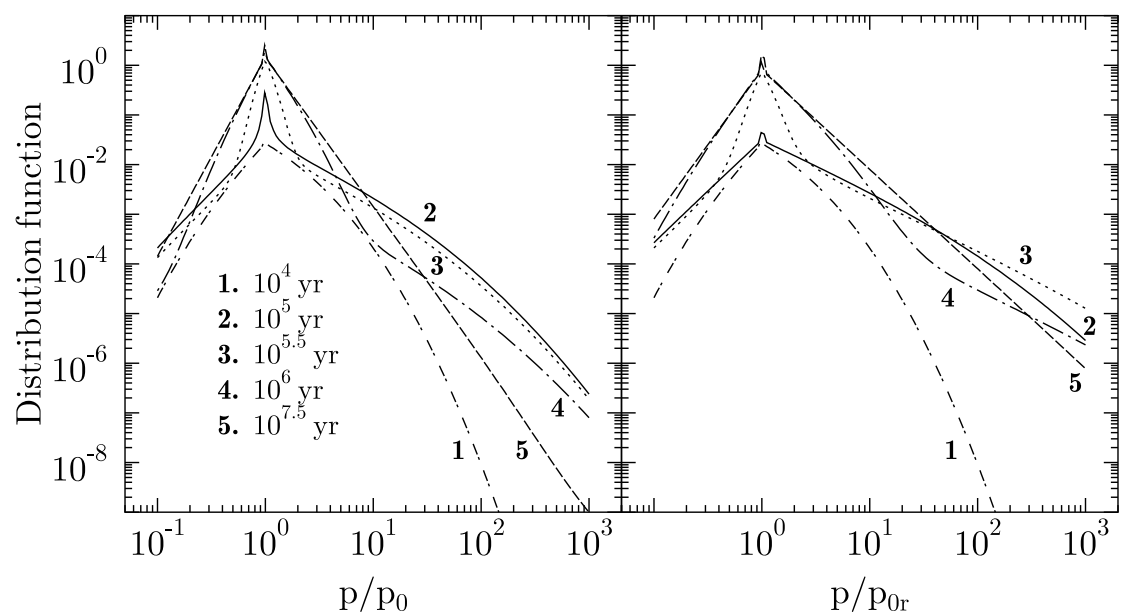

Figure 2. The temporal evolution of the particle distribution function in the SB of $R=220 \mathrm{pc}$, with the standard IMF. Monoenergetic injection was assumed with the injection energies $10 \mathrm{keV}$ (left panel) and $10 \mathrm{GeV}$ (right panel).

(2) A very important process of superthermal nuclei injection might be associated with fast moving knots and filaments very highly enriched with oxygen and other star burning products due to explosions of massive stars (Bykov, 1995) observed in some SN remnants like CAS A, Puppis A etc. These filaments moving with typical velocities of about $1000-5000 \mathrm{~km} \mathrm{~s}^{-1}$ are the sources of injected nuclei of relatively low ionisation stages. Note that a neutral atom which is evaporated from a metal rich knot or a filament (even of a low velocity) and then ionized within the bubble will be picked up by supersonically moving magnetized plasma and injected into the acceleration. Also, high velocity grains formed in supernova ejecta have been considered (Ramaty and Lingenfelter, 1999, and references therein) as a source of cosmic ray metals injection.

Both injection processes are expected to inject metal-rich nonthermal component. The second process should dominate at the early stages of SB evolution during the first few million years and might contribute substantially later on. Since the particle acceleration time in a SB is about a few times $10^{5}$ years (Figure 1) one may expect to have a source of nonthermal nuclei with greatly enhanced fluxes of metals during such a period. Recent measurements of ${ }^{59} \mathrm{Ni}$ and ${ }^{59} \mathrm{Co}$ abundances in galactic cosmic rays by CRIS onboard the ACE mission indicated a long cosmic ray acceleration period of $\sim 10^{5}$ years after the nucleosynthesis (Wiedenbeck et al., 1999), which is in a good agreement with the SB model discussed.

It is important to note that the efficiency of SB energy (MHD) conversion to nonthermal component is about a factor of 1.5-4 higher during the earliest stage of SB evolution (the first 3-10 Myr depending on the SB scale). The energy injection from supernovae explosions into a SB is roughly time independent for about $5 \times$ $10^{7} \mathrm{yr}$ for the standard IMF (e.g., McCray and Kafatos, 1987), but an account 
of massive star winds contribution would increase the energy injection rate at the earliest stages. Thus the energy converted to CRs at the stage of WR stars and most massive SN explosions could be comparable to that of the CRs accelerated after the first $10 \mathrm{Myr}$ in the SB model. This results in the enhancement of the abundances of the elements produced by the most massive SNe and the WR stars in the accelerated nonthermal component. The ${ }^{22} \mathrm{Ne}$ and ${ }^{12} \mathrm{C}$-rich $\mathrm{CR}$ component (e.g., Cassé and Paul, 1982; Maeder and Meynet, 1993; Meyer et al., 1997; Meynet et al., this volume) could be naturally accounted for in the SB model. The injection mechanism (2) - due to local ionization of the neutral atoms evaporating from knots and filaments ejected by a $\mathrm{SN}$ - can explain observed $A / Q$ enhancement (see, e.g., Meyer et al., 1997, for detailed analysis of observations), because of the low ionisation stage of the fast moving atoms evaporated from the metal rich knots. The quantitative prediction of CR abundances expected in the SB model depends on the details of the structure of SN ejecta. Complex kinetics of material mixing and condensation in a highly nonequilibrium SNR condition is not well established at the moment to model the structure of SN ejecta. A growing body of high resolution observations of SNRs with ISO, Chandra, and XMM, as well as high-quality optical data (e.g., Blair et al., 2000) is indicating complex structure of SN fast-moving debris of nuclear-processed material.

Later on an extended SB should be mostly a source of the standard cosmic rays (Axford, 1992; Bykov and Fleishman, 1992; Higdon et al., 1998) with the nuclei injection processes discussed above. The SB thermal plasma composition is close to the standard one at that stage, with possible excess of ${ }^{22} \mathrm{Ne}$, etc. in the nonthermal component accelerated at the previous stage. The ISM dust grains are also contributed like in the scenario of galactic CRs acceleration by isolated SNR shocks developed by Meyer et al. (1997) and Ellison et al. (1997).

\section{Nonthermal Processes in Supershells}

SBs might manifest themselves as a class of galactic objects with greatly enhanced fluxes of nonthermal nuclei with a non-standard composition. At a certain stage of their evolution, depending also upon the environmental conditions (which are different for early galaxies) SBs could be treated as sources of low-energy nonthermal nuclei. Nucleosynthesis and spallation reactions due to interactions of accelerated nonthermal nuclei with the ambient medium would be an efficient source of light elements (Cassé et al., 1995; Bykov, 1995; Ramaty et al., 1996, 1997, 2000; Duncan et al., 1997; Vangioni-Flam et al., 1998; Parizot and Drury, 1999; Fields et al., 2000; Parizot, 2000; Vangioni-Flam and Cassé, 2000). These reactions can drastically change the isotope composition in the supershell surrounding a SB which makes them responsible for variations of some isotope ratios observed in the ISM (e.g., Bykov, 1995). Due to diffusion of the nonthermal nuclei in the dense shell the scale of the variations could be as small as a few parsecs. Then the 'starformation 


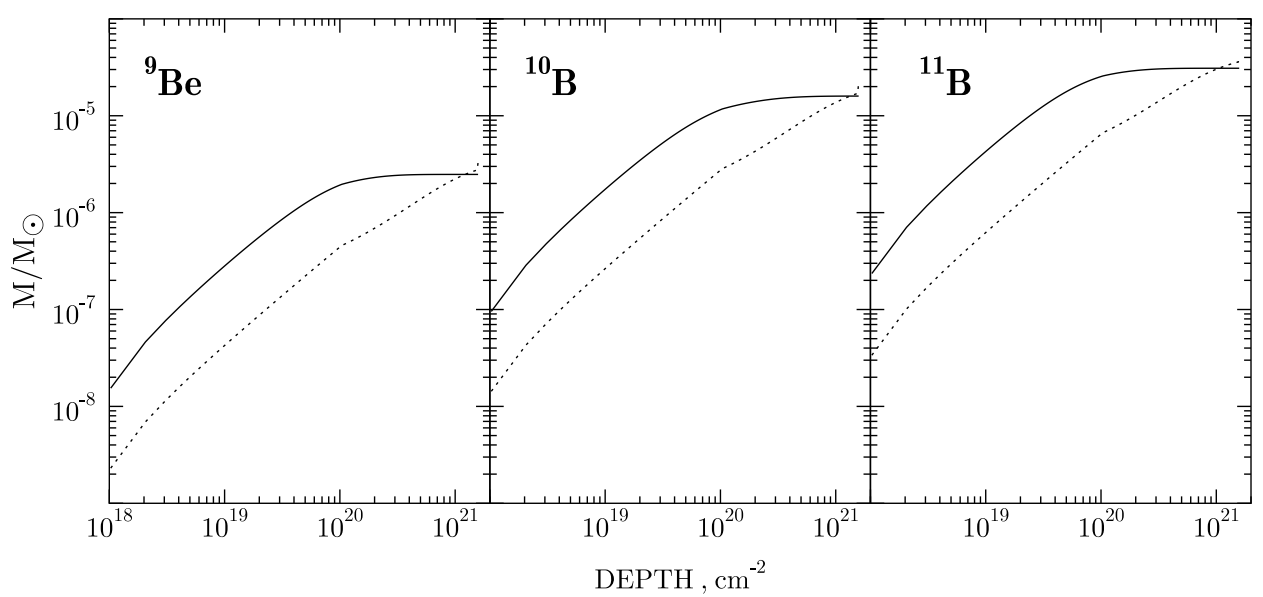

Figure 3. The light element deposition as a function of the supershell depth calculated for two different diffusion regimes (Bykov et al., 1999) (see text). The supershell surrounds a SB of the radius $220 \mathrm{pc}$.

wave' is able to reflect the abundance variations in the next generation of stars. It is important that being supplied with a source of violent MHD motions from SNe explosions in a SB a supershell should have MHD turbulence during at least 30 Myr. The reacceleration of nuclei inside a dense supershell is an important effect to compensate strong Coulomb losses. Modeling of light element production in such supershells with account of reacceleration effect has been performed by Bykov et al. (1999). In Figure 3 we presented a model result for production of ${ }^{9} \mathrm{Be}$, ${ }^{10} \mathrm{~B},{ }^{11} \mathrm{~B}$ as a function of the supershell depth. Diffusive propagation of fast nuclei in the shell is described by models with coefficients $\kappa_{0}=3 \times 10^{25} \mathrm{~cm}^{2} \mathrm{~s}^{-1}$ (solid line) and $\kappa_{0}=3 \times 10^{27} \mathrm{~cm}^{2} \mathrm{~s}^{-1}$ (dotted line) at GeV/nucl energy. These models account for the cases of strong and moderate scattering rates of nuclei by MHD waves, respectively.

Nuclear interaction lines are a natural test for observational diagnostic of the nonthermal nuclei component. Having in mind the efficiency of power conversion from the nonthermal nuclei to $\gamma$-ray lines to be typically below one percent, the source has to be rather nearby to be observed with the current instruments. Because of the temporal evolution of nonthermal particle spectra and composition in a SB one may expect the $\gamma$-ray line spectrum to be dominated by broad lines at the early stage of the evolution while narrow lines should dominate the late evolution stages.

\section{Acknowledgements}

It is a great pleasure for me to devote the paper to the 70th birthday of I.N.Toptygin. I thank the workshop organizers for their most kind hospitality. This work was sup- 
ported in part by INTAS 96-0390, INTAS-ESA 99-1627 and Fundamental Studies Program (Russian Universities) 015.02.01.01 grants.

\section{References}

Axford, W. I.: 1992, in G. P. Zank and T. K. Gaisser (eds.), 'Particle Acceleration on Galactic Scales', Particle Acceleration in Cosmic Plasmas, AIP Conf. Proc. 264, 45-56.

Blair, W. P. et al.: 2000, 'Hubble Space Telescope Observations of Oxygen-Rich Supernova Remnants in the Magellanic Clouds II', Astrophys. J. 537, 667-689.

Blitz, L.: 1993, in E. H. Levy and J. I. Lunine (eds.), 'Giant Molecular Clouds', Protostar and Planets III, University Arizona Press, Tucson, pp. 125-161.

Bykov, A. M.: 1988, 'A Model for the Generation of Interstellar Turbulence', Sov. Astron. Lett. 14, 60-63.

Bykov, A. M.: 1995, 'Nucleosynthesis from Nonthermal Particles', Space Sci. Rev. 74, 397-406.

Bykov, A. M.: 1999, in R. Ramaty et al. (eds.), 'Nonthermal Particles in Star Forming Regions', Li, Be, B, Cosmic Rays and Related X-and Gamma-Rays, ASP Conf. Series 171, 146-153.

Bykov, A. M. and Fleishman, G. D.: 1992, 'On Non-thermal Particle Generation in Superbubbles', Monthly Not. Roy. Astron. Soc. 255, 269-275.

Bykov, A. M. and Toptygin, I. N.: 1987, 'Effect of Shocks on Interstellar Turbulence and Cosmic-Ray Dynamics', Astrophys. Space Sci. 138, 341-354.

Bykov, A. M. and Toptygin, I. N.: 1993, 'Kinetics of Particle in the Strongly Turbulent Plasmas', Physics Uspekhi 36, 1020-1052.

Bykov, A. M, Gustov, M. Yu., and Petrenko, M. V.: 1999, in R. Diehl and D. Hartman (eds.), 'Energetic-Nuclei Acceleration and Interactions in the Early Galaxy', Astronomy with Radioactivities, MPE Report 274, 241-248.

Cassé, M., Lehoucq, R., and Vangioni-Flam, E.: 1995, 'Production and Evolution of Light Elements in Active Star-Forming Regions', Nature 373, 318-321.

Cassé, M. and Paul, J.: 1982, 'On the Stellar Origin of the ${ }^{22}$ Ne Excess in Cosmic Rays', Astrophys. J. 258, 860-863.

Chen, C. H., Chu, Y. H., Gruendl, R. A., and Points, S. D.: 2000, 'Hubble Space Telescope Wide Field Planetary Camera 2 Imaging of Shocks in Superbubbles', Astron. J. 119, 1317-1324.

Duncan, D. et al.: 1997, 'The Evolution of Galactic Boron and the Production Site of the Light Elements', Astrophys. J. 488, 338-349.

Ellison, D. C., Drury, L. O'C., and Meyer, J. P.: 1997, 'Galactic Cosmic Rays from Supernova Remnants - II', Astrophys. J. 487, 197-217.

Fields, B. D., Olive, K. A., Vangioni-Flam, E., and Cassé, M.: 2000, 'Testing Spallation Processes With Beryllium and Boron', Astrophys. J. 540, 930-945.

Heiles, C.: 1998, 'Whence the Local Bubble', Astrophys. J. 498, 698-703.

Higdon, J. C., Lingenfelter, R. E., and Ramaty, R.: 1998, 'Cosmic Ray Acceleration from Supernova Ejecta in Superbubbles', Astrophys. J. 509, L33-L36.

Korpi, M. J., Brandenburg, A., Shukurov, A., and Tuominen, I.: 1999, 'Evolution of a Superbubble in a Turbulent, Multi-phased and Magnetized ISM', Astron. Astrophys 350, 230-239.

McCray, R. and Kafatos, M.: 1987, 'Supershells and Propagating Star Formation', Astrophys. J. 317, 190-196.

Maeder, A., Meynet, G.: 1993, 'Isotopic Anomalies in Cosmic Rays and the Metallicity Gradient in the Galaxy', Astron. Astrophys. 278, 406-414.

Meyer, J. P., Drury, L. O'C., and Ellison, D. C.: 1997, 'Galactic Cosmic Rays from Supernova Remnants - I', Astrophys. J. 487, 182-196. 
Parizot, E.: 1998, 'The Orion Gamma-ray Emission and the Orion-Eridanus Bubble', Astron. Astrophys. 331, 726-736.

Parizot, E.: 2000, 'Superbubbles and the Galactic Evolution of Li, Be, B', Astron. Astrophys. (in press).

Parizot, E. and Drury, L.: 1999, 'Superbubbles as the Source of ${ }^{6} \mathrm{Li}, \mathrm{Be}$ and B in the Early Galaxy', Astron. Astrophys. 349, 673-684.

Ramaty, R. and Lingenfelter, R. E.: 1999, in R. Ramaty et al. (eds.), 'Spallogenic Light Elements and Cosmic Ray Origin', Li, Be, B, Cosmic Rays and Related X-and Gamma-Rays, ASP Conf. Series 171, 104-117.

Ramaty, R., Kozlovsky, B., and Lingenfelter, R. E.: 1996, 'Light Isotopes, Extinct Radioisotopes and Gamma-Ray Lines from Low-Energy Cosmic-Ray Interactions', Astrophys. J. 456, 525-540.

Ramaty, R., Kozlovsky, B., Lingenfelter, R. E., and Reeves, H.: 1997, 'Light Elements and Cosmic Rays in the Early Galaxy', Astrophys. J. 488, 730-748.

Ramaty, R., Scully, S. T., Lingenfelter, R. E., and Kozlovsky, B.: 2000, 'Light-Element Evolution and Cosmic-Ray Energetics', Astrophys. J. 534, 747-756.

Seo, E. S. and Ptuskin, V. S.: 1994, 'Stochastic Reacceleration of Cosmic Rays in the Interstellar Medium', Astrophys. J. 431, 705-714.

Tomisaka, K.: 1998, 'Superbubbles in Magnetized Interstellar Media', Monthly Notices Roy. Astron. Soc. 298, 797-810.

Vangioni-Flam, E. and Cassé, M.: 2000, 'LiBeB Production and Associated Astrophysical Sites', astro-ph/0001474.

Vangioni-Flam, E., Ramaty, R., Olive, K., and Cassé, M.: 1998, 'Testing the Primary Origin of Be and B in the Early Galaxy', Astron. Astrophys. 337, 714-720.

Wiedenbeck, M. E. et al.: 1999, 'Constraints on the Time Delay between Nucleosynthesis and Cosmic-Ray Acceleration', Astrophys. J. 523, L61-L64.

Woosley, S. E. and Weaver, T. A.: 1995, 'The Evolution and Explosion of Massive Stars II', Astrophys. J. Suppl. 101, 181-235. 